\title{
The effect of leptin and resveratrol on JAK/STAT pathways and Sirt-1 gene expression in the renal tissue of ischemia/ /reperfusion induced rats
}

\author{
Erkasap $\mathrm{S}^{1}$, Erkasap N², Bradford B ${ }^{3}$, Mamedova L ${ }^{3}$, Uysal $\mathrm{O}^{4}$, Ozkurt M², Ozyurt R², \\ Kutlay $\mathrm{O}^{2}$, Bayram $\mathrm{B}^{5}$ \\ Department of General Surgery, Medical Faculty, Eskisehir Osmangazi University, Eskisehir, Turkey. \\ nerkasap@gmail.com
}

\begin{abstract}
OBJECTIVE: Our study aimed to investigate the possible modifying effects of leptin and combined use of resveratrol on rat renal I/R injury and their relationship on signal pathways and apoptosis-related mechanisms. BACKGROUND: Renal ischemia-reperfusion (I/R) injury is an important cause of acute renal failure. METHODS: Male Sprague Dawley rats were divided into 5 groups: Control, I/R, I/R+leptin, I/R+resveratrol and $\mathrm{l} / \mathrm{R}+$ leptin+resveratrol. Leptin $(10 \mu \mathrm{g} / \mathrm{kg} \mathrm{BW})$ was administered (i.p.) $30 \mathrm{~min}$ prior to $\mathrm{l} / \mathrm{R}$. Resveratrol was administered by gavage at $20 \mathrm{mg} / \mathrm{kg} \mathrm{BW}$ per $\mathrm{d}$ for $12 \mathrm{~d}$ prior to I/R. The left renal artery was exposed to $1 \mathrm{~h}$ of ischemia and $1 \mathrm{~h}$ of reperfusion.

RESULTS: Resveratrol treatment alone increased TNF- $\alpha$, TNF- $\alpha$ R1, NF-KB, SIRT-1, STAT1 and STAT3 mRNA levels and decreased caspase 3 protein levels. Leptin treatment alone significantly decreased the caspase 3 protein levels. The combined use of resveratrol and leptin significantly increased STAT3, and caspase 3 mRNA levels, and decreased the caspase 3 protein levels. Apoptosis was significantly decreased especially in the leptin and leptin+resveratrol groups.

CONCLUSION: The present study suggest that a combined use of resveratrol and leptin has preventive and regulatory effects on renal I/R injury; the mechanism involves decreasing apoptosis, likely by altering the JAK/ STAT pathway and SIRT1 expression (Fig. 8, Ref. 24). Text in PDF www.elis.sk. KEY WORDS: leptin, resveratrol, TNF-alpha, caspase 3, apoptosis.
\end{abstract}

\section{Introduction}

Leptin, a protein hormone that contains 167 amino acids, is known to induce many metabolic effects via the JAK/STAT signaling pathway. Our previous studies reported that leptin has a protective effect on renal I/R model induced by 1 hour of ischemia following 1 hour of reperfusion (1). Another study showed the protective effects of resveratrol against oxidative stress on cardiac I/R model in the same time periods (2). Smith et al investigated the role of the JAK/STAT pathway in the cardioprotective effects of resveratrol and suggested that the effects may involve

${ }^{1}$ Department of General Surgery, Medical Faculty, Eskisehir Osmangazi University, Eskisehir, Turkey, ${ }^{2}$ Department of Physiology, Medical Faculty, Eskisehir Osmangazi University, Eskisehir, Turkey, ${ }^{3}$ Department of Animal Sciences and Industry, Kansas State University, USA, ${ }^{4}$ Cellular Therapy and Stem Cell Production, Application and Research Center ESTEM, Eskisehir Osmangazi University, Eskisehir, Turkey, and ${ }^{5}$ Medical Laboratory Skills, Vocational School of Health Services, Muğla Sitki Koçman University, Mugla, Turkey

Address for correspondence: N. Erkasap, Prof Dr, Department of Physiology, Medical Faculty, Eskisehir Osmangazi University, Eskisehir, Turkey.

Acknowledgement: The research was supported by a grant (no: 2013109) from the Eskisehir Osmangazi University Scientific Research Projects Committee, Turkey. leptin-induced STAT3 phosphorylation (3). Resveratrol, a natural polyphenol compound, has many biochemical and physiological effects, including anti-inflammatory, antioxidant, antiapoptotic and antiproliferative effects (2). Recent studies have suggested that resveratrol may protect against ischemic damage in skeletal muscle, heart and brain $(2,4)$. Nevertheless, the molecular mechanisms of resveratrol action are not yet clarified. The in vivo antioxidant and anti-inflammatory effects of resveratrol may be mediated by Sirtuin-1 (SIRT-1) (5). Similar to resveratrol, leptin has antioxidant, antiapoptotic and anti-inflammatory effects and causes an increase in SIRT1 gene expression (6). Although in vitro studies have reported that resveratrol may abrogate acute stress by increasing SIRT1 gene expression $(5,7)$, there are no in vivo studies, to our knowledge, on SIRT1 gene expression in renal $\mathrm{I} / \mathrm{R}$ injury.

TNF- $\alpha$, a proinflamatory cytokine released by many cell types, plays a central role in ischemia-reperfusion ( $\mathrm{I} / \mathrm{R})$ injury. These roles are well presented in liver and myocardiac tissues $(8$, 9). It is also well known that TNF- $\alpha$ plays a critical role in many types of kidney damage and TNF- $\alpha$ Receptor 1 (TNF- $\alpha$ R1) is the key mediator of this role by causing apoptosis (10). TNF- $\alpha$ is induced both directly and indirectly by Nuclear factor- $\kappa \mathrm{B}$ (NF$\kappa B$ ) in inflamation occured in $\mathrm{I} / \mathrm{R}$ degeneration (11). Spandou et al (12) suggested that NF- $\mathrm{KB}$ activation may have a crucial role 
in the pathogenesis of several renal diseases. Our previous studies reported leptin treatment of the $\mathrm{I} / \mathrm{R}$ group caused a marked decrease in the TNF- $\alpha$ values compared to I/R alone in rat bladder tissues (13). Gualdoni et al showed that the use of resveratrol increased the level of TNF- $\alpha$ soon after application (14). However, the molecular mechanisms and interaction between TNF- $\alpha$ and these two agents at the level of cellular pathways have not been clarified.

The aim of this study is to investigate the possible modifying effects of acute leptin, chronic resveratrol and combined treatment on renal I/R injury-related molecular mechanisms such as TNF- $\alpha$, JAK/STAT SIRT1 pathways and their relation with apoptosis in rats.

\section{Method}

The Eskisehir Osmangazi University Institutional Local Animal Care and Use Committee (314/2012) approved all experimental procedures.

\section{Design and treatments}

A total of 40 male Sprague-Dawley rats weighing 200 $250 \mathrm{~g}$ and aged 3-4 months were randomly assigned to experimental group. All rats were anesthetized by an intraperitoneal (i.p.) injection of ketamine and xylazine. The study groups $(\mathrm{n}=8)$ were as follows: Group A: Control animals, group B: $\mathrm{I} / \mathrm{R}$, group $\mathrm{C}: \mathrm{I} / \mathrm{R}+$ leptin, group $\mathrm{D}: \mathrm{I} / \mathrm{R}+$ resveratrol, group $\mathrm{E}: \mathrm{I} /$ $\mathrm{R}+$ leptin+resveratrol. No surgical procedure was performed in control animals, saline was injected $60 \mathrm{~min}$ before anesthesia. Intraperitoneal saline was injected $60 \mathrm{~min}$ before the $\mathrm{I} / \mathrm{R}$ procedures. For rats in the I/R+leptin group, $10 \mu \mathrm{g} / \mathrm{kg}$ of leptin was given intraperitoneally to the animals $60 \mathrm{~min}$ before the $\mathrm{I} / \mathrm{R}$ procedure. For rats in the $\mathrm{I} / \mathrm{R}+$ resveratrol group, $20 \mathrm{mg} / \mathrm{kg}$ of resveratrol was given by oral gavage for 14 days before the I/R procedure. For rats in $\mathrm{I} / \mathrm{R}+$ leptin+resveratrol group, animals were treated with 20 $\mathrm{mg} / \mathrm{kg}$ of resveratrol given by oral route for 14 days, followed by an intraperitoneal injection of $10 \mu \mathrm{g} / \mathrm{kg}$ of leptin $60 \mathrm{~min}$ before the $\mathrm{I} / \mathrm{R}$ procedure.

\section{Surgical operation and sample collection}

Under aseptic conditions, a midline incision was performed in the ventral part of the body. In control group after 2 hours of anesthesia, kidneys and blood samples were withdrawn. In I/R groups, a non-traumatic vascular clamp was applied to the left renal artery to interrupt the blood flow for $60 \mathrm{~min}$ (ischemia), followed by removal of the clamp for $60 \mathrm{~min}$ (reperfusion). At the end of the reperfusion period, the animals were euthanized, blood samples and left kidney were collected. The I/R procedure and the post-I/R procedures were performed in B, C, D, E groups.

\section{RNA Extraction and Real-Time PCR}

Total RNA was extracted from approximately $100 \mathrm{mg}$ of tissue using tripure solution according to the manufacturer's instructions (Roche, Mannheim, Germany). RNA concentration and purity were analyzed by measuring the optical density at $260 \mathrm{~nm}$ (Nano-
Drop 1000, Thermo Scientific, USA). One microgram of RNA was converted to cDNA using cDNA Synthesis Kit (Roche Nano Lightcycler Roche Diagnostics, Mannheim, Germany) according to the manufacturer's instructions. The abundance of TNF- $\alpha$, TNF- $\alpha$ R1, NF- $\kappa$ B, SIRT-1, STAT1, STAT3 and caspase 3 mRNA were analyzed according to the manufacturer's instructions, using the beta-actin as a reference gene and Roche Nano Lightcycler (Roche Diagnostics, Mannheim, Germany).

\section{Protein isolation and western blotting}

Total protein was extracted from approximately $100 \mathrm{mg}$ of tissue using tripure solution according to the manufacturer's instructions (Roche). The protein concentration was measured using spectroscopy (NanoDrop). Then, 80 - $\mu$ gr samples were placed into the wells of $10 \%$ SDS-Polyacrylamide gels to separate the samples into bands by their molecular weight by using electrophoresis. After completing the electrophoresis, the gels were transferred to nitrocellulose membranes (Bio-Rad CA, USA) and incubated with rabbit anti-caspase 3 antibodies (1:1,000 dilution; Santa Cruz Biotechnology, Santa Cruz, CA). The secondary antibody (antirabbit IgG, Santa Cruz Biotechnology) was diluted 10,000-fold. All incubations were performed in Tris- $\mathrm{HCl}$ buffer, $\mathrm{pH} 7.5$, with 0.1 $\%$ Tween 20 and $5 \%$ dry milk. Dry milk was not added for the final washing. Immunodetection was performed using chemiluminescence (West-Dura, Thermo Scientific, Waltham, MA). The signal intensity was determined by densitometry (ChemiDoc-It Imaging System, UVP Inc., Upland, CA), and the means and SDs were calculated for each tissue type.

\section{DNA nick end-labeling of tissue sections}

For the detection of apoptosis in tissues, a TUNEL-based apoptosis kit (Cat. no. S7101; ApopTag Plus Peroxidase Kits, Chemicon International, USA) was used. In this method, the sections were first deparaffinized and rehydrated. They were then permeabilized with proteinase $\mathrm{K}$ and endogen peroxidase, inactivated by $10 \% \mathrm{H}_{2} \mathrm{O}_{2}$. For DNA labeling, Tdt Labeling reaction mix and Tdt Enzyme mixture were used. Following incubation, the reaction was detected with conjugate and diaminobenzidine (DAB) solution in $\mathrm{H}_{2} \mathrm{O}_{2}$ /urea mixture. Methyl green (3\%) was used for counterstaining. The slides were then dehydrated and mounted. The TUNEL-positive brown-colored cells were considered to be apoptotic cells, in agreement with the positive control supplied by the manufacturer.

\section{Statistical analysis}

Transcript data were expressed relative to the control (set to 1) \pm standard. Statistical analysis was performed by using the GraphPad software program. Following the determination of TNF- $\alpha$, TNF- $\alpha$ R1, NF- $\kappa$ B, SIRT-1, STAT1, STAT3 and Caspase 3 mRNA gene expression using beta-actin as a reference gene, the data obtained from RT PCR were calculated using the formula $2^{-\Delta \Delta C t}$. The WB results were analyzed by using one-way ANOVA (Tukey's test). Histological results were analyzed using the non-parametric Krusskal Wallis test and the intergroup differences were analyzed by Dunn's test. 


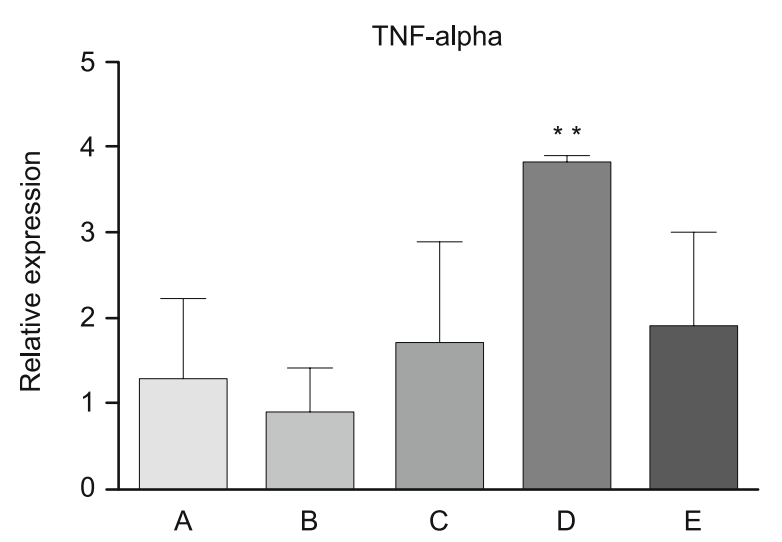

Fig. 1A. TNF-alfa mRNA expression. Resveratrol treatment group (D), compared to the control (A) group; ** $\mathrm{p}<0.01$ (One Way ANOVA/Tukey).

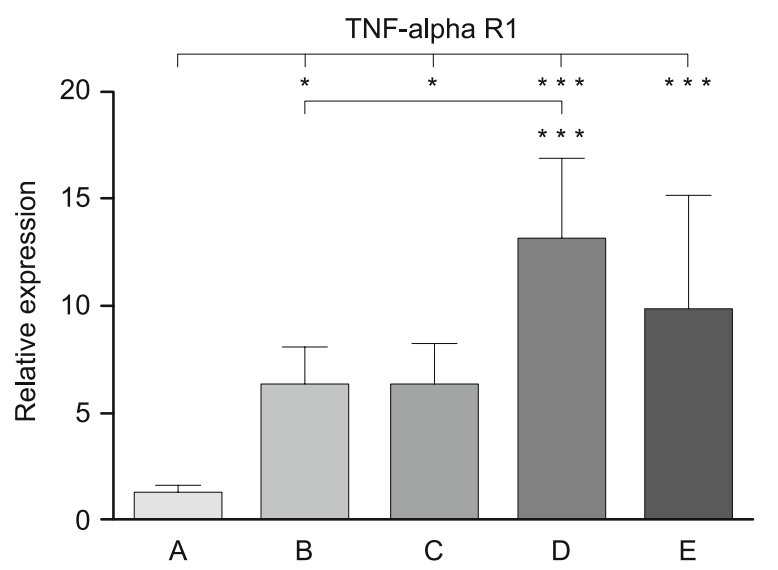

Fig. 1B. TNF-alfa R1 mRNA expression. Control (A) group compared to I/R (B) group; * $p<0.05$ (One Way ANOVA/Tukey), Control (A) group compared to the leptin treatment (C) group; * $p<0.05$ (One Way ANOVA/Tukey), Control (A) group compared to the resveratrol treatment (D) and leptin+resveratrol treatment (E) groups; *** $\mathbf{p}<$ 0.001 (One Way ANOVA/Tukey). I/R (B) group compeared to C group; $* \mathbf{p}<0.05$ (One Way ANOVA/Tukey), and compeared to D, E; *** p $<0.001$ (One Way ANOVA/ Tukey).

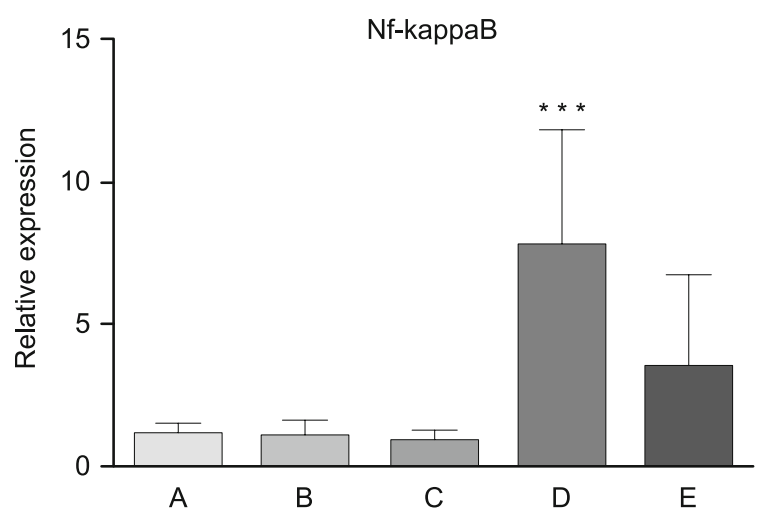

Fig. 2. NF-kB mRNA expression. Resveratrol treatment (D) group compared to the control (A) group; $* * * \mathbf{p}<0.001$ (One Way ANOVA/ Tukey).

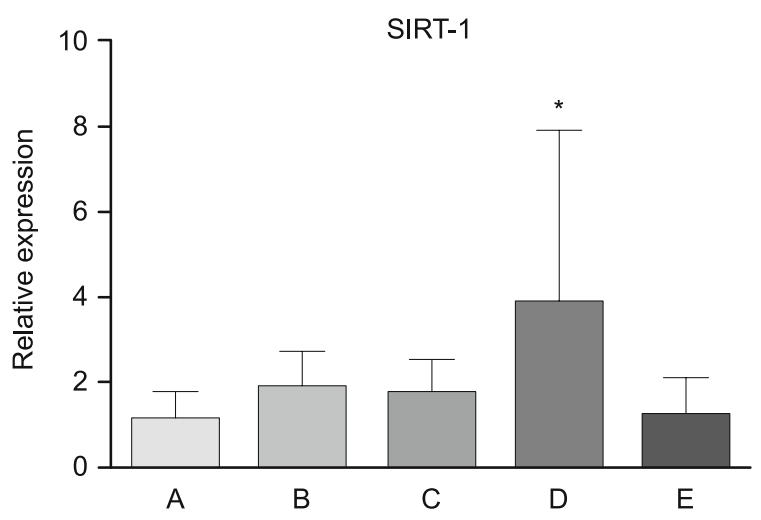

Fig. 3. SIRT-1 mRNA expression. Resveratrol treatment group (D) compared to the control (A) group; * $p<0.05$ (One Way ANOVA/ Tukey).

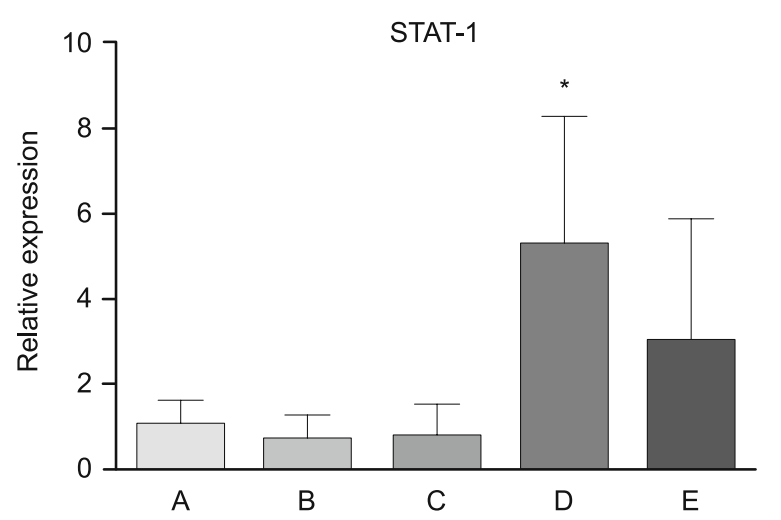

Fig. 4. STAT1 mRNA expression. Resveratrol treatment (D) group compared to the control (A) group; * $p<0.05$ (One Way ANOVA/ Tukey).

\section{Results}

\section{Real-time PCR}

TNF- $\alpha$ mRNA abundance was not significantly different in the leptin group but was significantly higher in the resveratrol group compared with the control group ( $p<0.01$, A vs D) (Fig. 1A). TNF- $\alpha$ R1 mRNA abundance was significantly increased in all groups compared with the control group $(\mathrm{p}<0.05 \mathrm{~A}$ vs $\mathrm{B}$, C, $p<0.001$ A vs D, E) (Fig. 1B). Similar to TNF- $\alpha$, the NF$\kappa B$ mRNA abundance was significantly higher in the resveratrol group than in the control group ( $p<0.001$ A vs D) (Fig. 2). SIRT1 mRNA abundance was significantly higher in the resveratrol group than in the control group ( $<<0.05 \mathrm{~A}$ vs D) (Fig. 3). The STAT1 mRNA abundance measured by RT-PCR was significantly higher in the resveratrol group compared with the control group ( $p<0.05$, A vs D) (Fig. 4). STAT3 mRNA abundance increased significantly in the resveratrol and leptin+resveratrol groups compared with the control group ( $p<0.05 \mathrm{~A}$ vs $\mathrm{D}, \mathrm{p}<0.01 \mathrm{~A}$ vs E) (Fig. 5). Caspase3 mRNA abundance was significantly higher in 


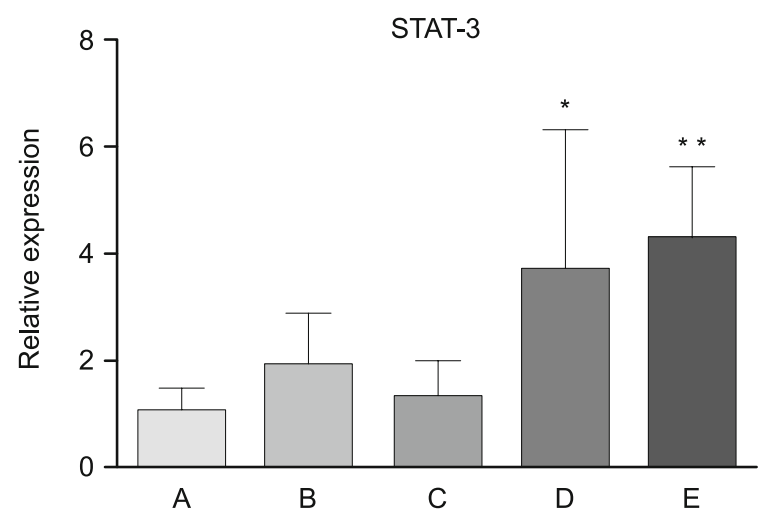

Fig. 5. STAT3 mRNA expression. Resveratrol treatment (D) group compared to the control (A) group; * $p<0.05$ (One Way ANOVA/ Tukey), and leptin+resveratrol treatment (E) group compared to the control (A) group ** p < 0.01 (One Way ANOVA/Tukey).

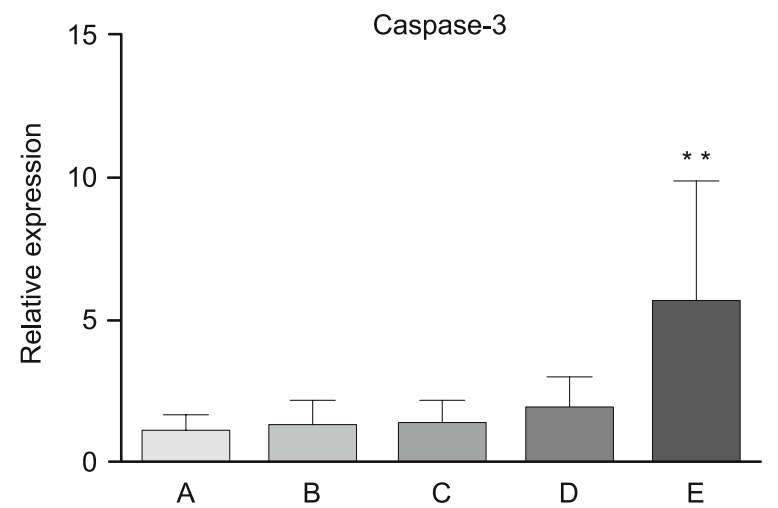

Fig. 6A. Caspase 3 mRNA expression. Leptin+resveratrol treatment (E) group compared to the control (A) group ** p $<0.01$ (One Way ANOVA/Tukey).

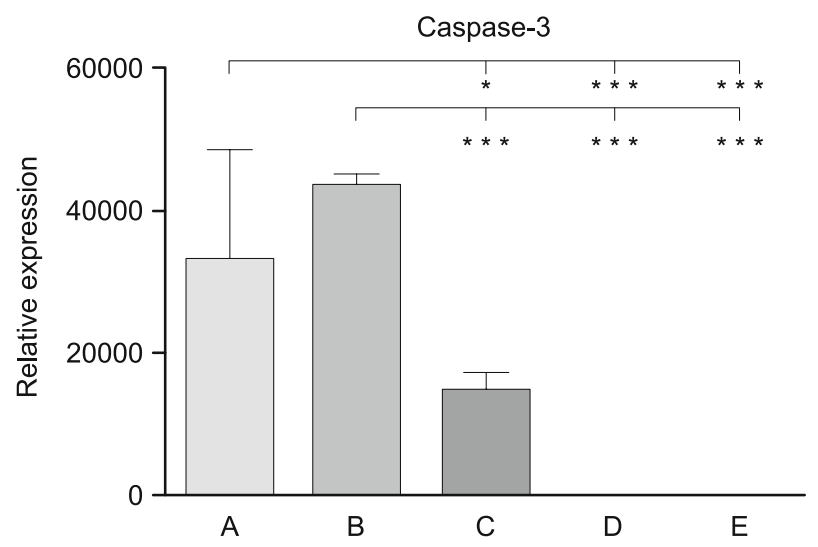

Fig. 6B. Caspase 3 protein level. Control (A) group compared to the leptin treatment (C) group; * $p<0.05$ (One Way ANOVA/Tukey), Control (A) group compared to the resveratrol treatment (D) and leptin+resveratrol treatment (E) groups; *** p $<0.001$ (One Way ANOVA/Tukey). I/R (B) group compeared to all the treatment groups (C, $\mathrm{D}, \mathrm{E}) ; * * * \mathrm{p}<0.001$ (One Way ANOVA/Tukey).

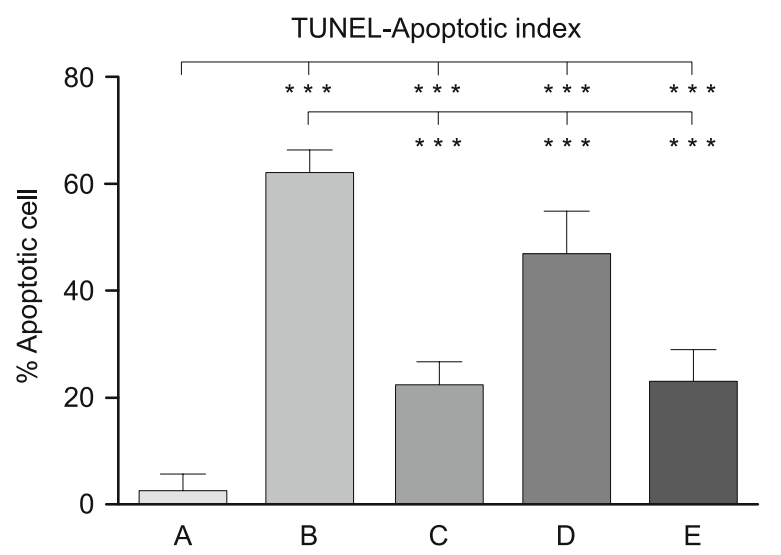

Fig. 7. TUNEL staining and apoptotic index. Control (A) group compared to all of $(\mathrm{B}, \mathrm{C}, \mathrm{D}, \mathbf{E})$ groups; $* * * \mathbf{p}<\mathbf{0 . 0 0 1}$ (Kruskall Wallis/ Dunns) $I / R$ (B) group compared to all of the treatment $(C, D, E)$ groups; $* * * \mathbf{p}<\mathbf{0 . 0 0 1}$ (Kruskall Wallis/ Dunns).

the leptin+resveratrol than in the control group $(\mathrm{p}<0.01 \mathrm{~A}$ vs $\mathrm{E})$ (Fig. 6A).

\section{Western Blot}

The caspase 3 protein level significantly decreased in the leptin, resveratrol and leptin+resveratrol groups compared with the control group ( $p<0.05 \mathrm{~A}$ vs $\mathrm{C}, \mathrm{p}<0.001 \mathrm{~A}$ vs $\mathrm{D}, \mathrm{E}$ and $\mathrm{B}$ vs C, D, E) (Fig. 6B).

\section{Histological analysis results}

For the calculation of apoptotic index, TUNEL (+) tubular epithelial cells were counted in 10 randomly selected fields under 20X magnification. Histological evaluation of apoptosis detected by TUNEL staining revealed a marked apoptosis in the I/R group, which was decreased significantly in all of the treatment groups but had a more pronounced effect in the leptin and leptin+resveratrol groups ( $\mathrm{p}<0.001$, B vs C, D, E) (Figs 7 and 8A-E).

\section{Discussion}

The results of the present study showed that renal tissue injury was modified in the leptin and leptin+resveratrol groups by keeping the TNF- $\alpha$ mRNA expression similar to that of the control group. Ghanim has reported that TNF- $\alpha$ levels were increased shortly after resveratrol administration (15). It is parallel in our study; we also measured increased TNF-alpha levels just after resveratrol treatment. TNF- $\alpha$ R1 mRNA level significantly increased in all groups compared to control group. However, this increase was impressive in resveratrol and leptin+resveratrol groups. These all results suggest that the use of resveratrol alone increases the levels of TNF- $\alpha$ soon after application, however the combined use of resveratrol with leptin modifies the tissue injury by decreasing the TNF- $\alpha$ level to the control levels. NF- $\kappa B$, signaling is described as one of the major transcriptional regulators of TNF- $\alpha$ expression (16). Gualdoni et al have also reported that treatment with resveratrol increases NF- $\mathrm{\kappa B}$ expression in human 

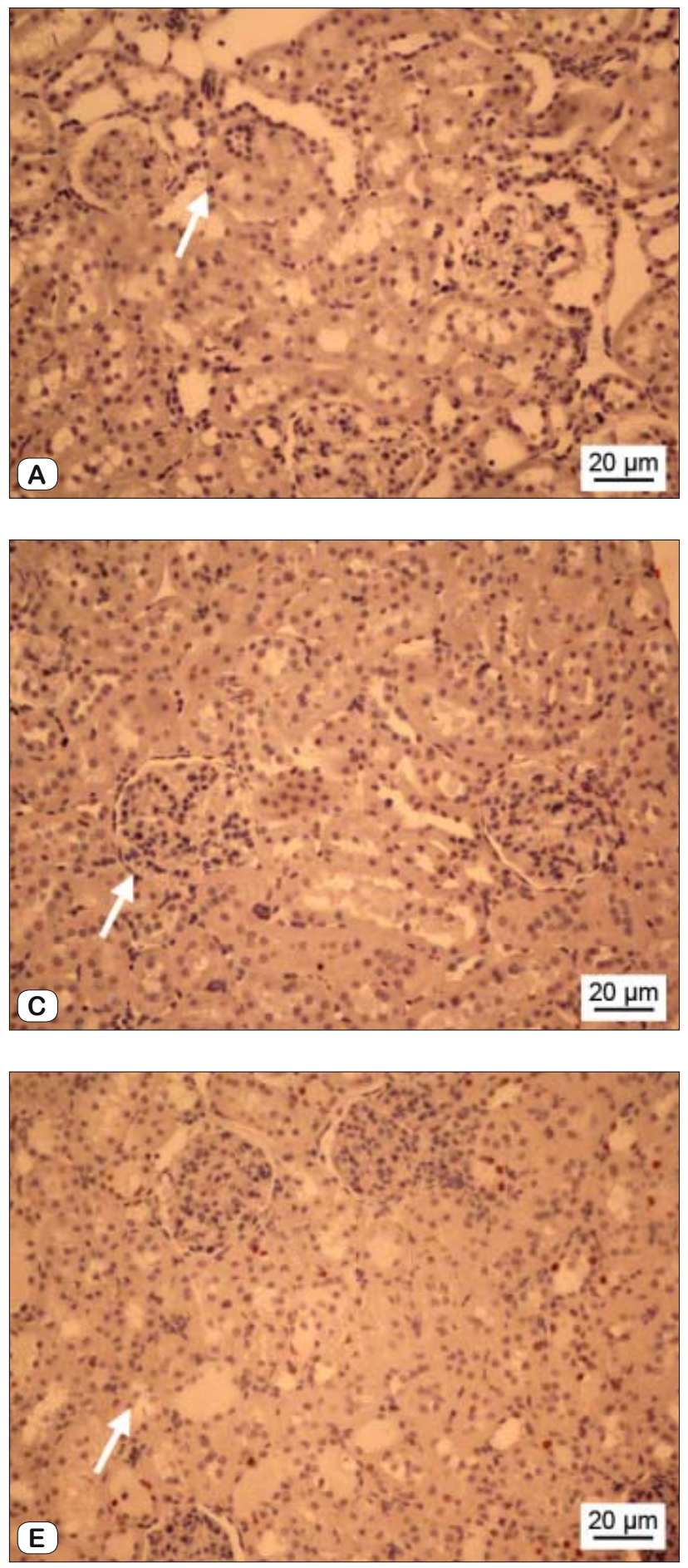

monocytes in a dose-dependent manner (14). In our study, parallel to TNF- $\alpha$ mRNA level, NF- $\kappa B$ mRNA level also increased in resveratrol treated groups, indicating that enhanced TNF- $\alpha$ production in the groups was caused by triggering NF- $\kappa \mathrm{B}$, signaling. Resveratrol has antiapoptotic effects by increasing the expression of SIRT1 gene, a member of sirtuins (6). SIRT1 plays a pivotal role in regulation of NF- $\mathrm{BB}-\mathrm{dependent}$ proinflammatory media-
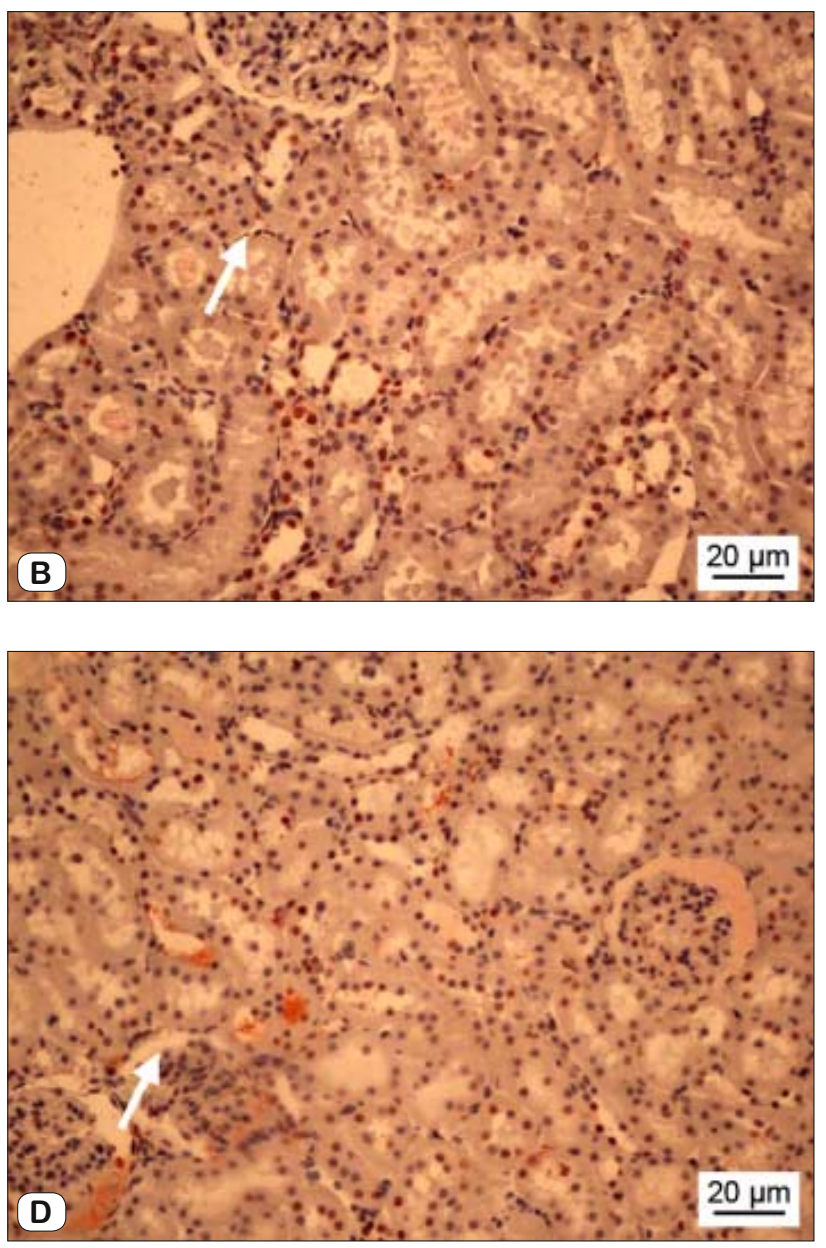

Fig. 8. TUNEL staining and apoptotic index. A) Control group; normal histological appearance; TUNEL, 20X, B) I/R group; brown-stained TUNEL (+) apoptotic epithelial nuclei, TUNEL, 20X, C) Leptin group; brown-stained TUNEL $(+)$ apoptotic epithelial nuclei, TUNEL, 20X, D) Resveratrol group; brown-stained TUNEL $(+)$ apoptotic epithelial nuclei, TUNEL, 20X, E) Leptin+resveratrol group; brown-stained TUNEL (+) apoptotic epithelial nuclei, TUNEL, 20X.

tor expression. Many studies in different tissues have shown that effects of resveratrol are via modulation of NF- $\mathrm{BB}(17)$. Although these effects have been confirmed in in vitro studies $(5,7)$, there are no in vivo studies investigating the role of SIRT1 gene expression in renal I/R injury. According to our study, we showed that SIRT1 mRNA expression was found to be increased in the resveratrol-treated group compared with the control and I/R groups in rats. Several $\mathrm{I} / \mathrm{R}$ studies demonstrated the contribution of the JAK/STAT pathway to injury in various tissues (18-20). In an in vitro study performed by Cao et al, a knockdown of STAT-1 gene expression decreased apoptosis induced by TNF- $\alpha$ and TNF- $\alpha$ also increased STAT-1 phosphorylation (21). Luna-Ortiz et al reported that TNF- $\alpha$ interact with JAK/STAT pathway and phosphorylates STAT-3 (22). Similar to those studies, our results suggest that the increase in TNF- $\alpha$ in resveratrol-treated group results in an increased JAK/STAT pathway activity via increasing STAT-1 and 
$443-448$

STAT-3 mRNA expression. Smith et al reported the role of the JAK/STAT pathway in the cardioprotective effects of resveratrol and suggested that the effects may involve leptin-induced STAT3 phosphorylation (3). In our study also, the combined use of resveratrol with leptin modifies the tissue injury by leptin-induced STAT3 phosphorylation. Caspase 3 is a key enzyme that leads to apoptosis in various cell types (23). According to control group, although there was a small increase in caspase 3 protein expression in sham group, but this elevation was not significant. Compared to similar studies we surmise that this nonsignificant result was due to a shorter reperfusion period (24). As consistent with apoptotic evaluation, renal caspase 3 protein expression was found to be decreased significantly in the leptin-treated group. Conversely, caspase 3 mRNA expression was found to be increased in the leptin+resveratrol group compared with the control group. It may suggest that although there is sufficient caspase 3 mRNA expression, the translation is defective, i.e., caspase 3 is inhibited at the protein synthesis level. However, according to TUNEL staining, apoptosis was significantly more inhibited by leptin than in resveratrol and resveratrol + leptin treatment compared with the $I / R$ group. Leptin showed modulatory effect on renal I/R injury by decreasing apoptosis, possibly through regulatory effects of the JAK/STAT signaling pathway and SIRT1 gene expression. On base of our experimental data, we suggest that the future clinical use of leptin-based therapies may exert protective effects on renal ischemia reperfusion injury.

\section{References}

1. Erkasap S, Erkasap N, Koken T, Kahraman A, Uzuner K, Yazihan $\mathbf{N}$ et al. Effect of leptin on renal ischemia-reperfusion injury damage in rats. J Physiol Biochem 2004; 60: 79-84.

2. Dernek S, Ikizler M, Erkasap N, Ergun B, Koken T, Yilmaz K et al. Cardioprotection with resveratrol pretreatment: improved beneficial effects over standard treatment in rat hearts after global ischemia. Scand Cardiovasc J 2004; 38: 245-254.

3. Smith CCT, Dixon RA, Wynne AM, Theodorou L, Ong SG, Subrayan $\mathrm{S}$ et al. Leptin-induced cardioprotection involves JAK/STAT signaling that may be linked to the mitochondrial permeability transition pore. Am J Physiol Heart Circ Physiol 2010; 299: H1265-H1270.

4. Ikizler M, Ovali C, Dernek S, Erkasap N, Sevin B , Kaygisiz Z et al. Protective effects of resveratrol in ischemia-reperfusion injury of skeletal muscle: A clinically relevant animal model for lower extremity ischemia. Chin J Physiol 2006; 49 (4): 204-209.

5. Xu Y, Nie L, Yin YG, Tang JL, Zhou JY, Li DD et al. Resveratrol protects against hyperglycemia-induced oxidative damage to mitochondria by activating SIRT1 in rat mesangial cells. Toxicol Appl Pharmacol 2012; 259 (3): 395-401.

6. Avraham Y, Davidi N, Porat M, Chernoguz D, Magen I, Vorobeiv L et al. Leptin reduces infarct size in association with enhanced expression of CB2, TRPV1, SIRT-1 and leptin receptor. Curr Neurovasc Res 2010; 7: 136-43.

7. Hasegawa K, Wakino S, Yoshioka K, Tatematsu S, Hara Y, Minakuchi $\mathbf{H}$ et al. Sirt1 protects against oxidative stress-induced renal tubular cell apoptosis by the bidirectional regulation of catalase expression. Biochemical and Biophysical Research Communications 2008; 372: 51-56.
8. Perry BC, Soltys D, Toledo AH, Toledo-Pereyra LH. Tumor necrosis factor- $\alpha$ in liver ischemia/reperfusion injury. J Invest Surg 2011; 24 (4): $178-188$

9. Kleinbongard P, Schulz R, Heusch G. TNF $\alpha$ in myocardial ischemia/reperfusion, remodeling and heart failure. Heart Fail Rev 2011; 16 (1): 49-69.

10. Ernandez T, Mayadas TN. Immunoregulatory role of TNF alpha in inflammatory kidney diseases. Kidney Int 2009; 76 (3): 262-276.

11. Kinaci MK, Erkasap N, Kucuk A, Koken T, Tosun M. Effects of quercetin on apoptosis, NF- $\mathrm{\kappa B}$ and NOS gene expression in renal ischemia/ reperfusion injury. Exp Ther Med 2012; 3: 249-254.

12. Spandou E, Tsouchnikas I, Karkavelas G, Dounousi E, Simeonidou C, Guiba-Tziampiri $\mathbf{O}$ et al. Erythropoietin attenuates renal injury in experimental acute renal failure ischaemic/reperfusion model. Nephrol Dial Transplant 2006; 21: 330-336.

13. Hamarat M, Yenilmez A, Erkasap N, Isikli B, Aral E, Koken T et al. Protective effects of leptin on ischemia/reperfusion injury in rat bladder. Chinese Journal of Physiology 2010; 53(3): 145-150.

14. Gualdoni GA, Kovarik JJ, Hofer J, Dose F, Pignitter M, Doberer D et al. Resveratrol enhances TNF- $\alpha$ production in human monocytes upon bacterial stimulation. Biochim Biophys Acta 2014; 1840: 95-105.

15. Ghanim H, Sia CL, Korzeniewski K, Lohano T, Abuaysheh S, Marumganti A et al. A resveratrol and polyphenol preparation suppresses oxidative and inflammatory stress response to a high-fat, highcarbohydrate meal. J Clin Endocrinol Metab 2011; 96: 1409-1414.

16. Liu H, Sidiropoulos P, Song G, Pagliari LJ, Birrer MJ, Stein B et al. TNF-alpha gene expression in macrophages: regulation by NF-kappa $B$ is independent of c-Jun or C/EBP beta. J Immunol 2000; 164: 4277-4285.

17. Lappas M, Mitton A, Lim R, Barker G, Riley C, Permezel M. SIRT1 is a novel regulatory of key pathways of human labor. Biol Reprod 2011; 84: 167-178.

18. Bolli R, Dawn B, Xuan YT. Emerging role of the JAK-STAT pathway as a mechanism of protection against ischemia/reperfusion injury. J Mol Cell Cardiol 2001; 33: 1893-1896.

19. Bolli R, Dawn B, Xuan YT. Role of the JAK-STAT pathway in protection against myocardial ischemia/reperfusion injury. Trends Cardiovasc Med 2003; 13: 72-79.

20. Chung EY, Kim BH, Hong JT, Lee CK, Ahn B, Nam SY et al. Resveratrol down-regulates interferon- $\gamma$-inducible inflammatory genes in macrophages: molecular mechanism via decreased STAT-1 activation. J Nutr Biochem 2011; 22: 902-909.

21. Cao ZH, Zheng QY, Li GG, Hu X, Feng S, Xu G, Zhang K. STAT1mediated down-regulation of Bcl-2 expression is involved in IFN- $\gamma /$ TNF$\alpha$-induced apoptosis in NIT-1 cells. PLOS One 2014; 10 (3): 1-12.

22. Ortiz-Luna P, Torres JC, Pastelin G, Martinez-Rosas M. Myocardial postconditioning: anesthetic considerations. Arch Cardiol Mex 2011; 81 (11): 33-46.

23. Yu W, Fu YC, Wang W. Cellular and molecular effects of resveratrol in health and disease. J Cell Biochem 2012; 113: 752-759.

24. Yucel G, Ozsoy M, Kocak A, Ozkececi ZT, Karavelioglu A, Bozkurt MF, Cartill O, Keles I, Kocak H, Celik S. Antioxidant, Antiapoptotic and Inflammatory Effects of Interleukin-18 Binding Protein on Kidney Damage Induced by Hepatic Ischemia Reperfusion. Am J Med Sci 2016; 351 (6): 607-615.

Received March 23, 2017. Accepted April 24, 2017. 